\title{
O050: Antibiotics in 70 German intensive care units: risk factors for high overall consumption
}

\author{
S Schneider ${ }^{*}$, F Schwab, P Gastmeier, E Meyer \\ From 2nd International Conference on Prevention and Infection Control (ICPIC 2013) \\ Geneva, Switzerland. 25-28 June 2013
}

\section{Introduction}

Due to increasing burden of multidrug resistant organisms and the lack of new antimicrobial substances prudent use of antibiotics (AB) becomes more essential than ever before. Surveillance of antimicrobial use is an important component of $\mathrm{AB}$ stewardship.

\section{Objectives}

To explore, whether there are certain risk patterns in intensive care units (ICUs) determining the total $A B$ use.

\section{Methods}

We analysed the $A B$ usage of 70 German ICUs in the year 2011. The data were collected in a standardized way within the SARI (Surveillance of Antimicrobial Use and Antimicrobial Resistance in ICUs) system. AB use densities (AD) were calculated as daily defined doses (DDD) per 1000 patient days (pd) for all systemically applied substances. We performed a stepwise forward multivariable analysis for the total $\mathrm{AB}$ use as binary outcome (total $\mathrm{AD}<=$ or $>75$. percentile). The following variables were included: hospital type (university, teaching, other), hospital size ( $<=$ or $>600$ beds), ward type (interdisciplinary, surgical, medical ICU), ward size $(<=$ or $>12$ beds), number of used $A B$ substances, number of used $A B$ groups, $A D s$ of $A B$ treatment groups and fractions of most used $A B$ groups.

\section{Results}

The median number of $\mathrm{AB}$ substances was 28 (range 1036 ), the median number of $A B$ groups was 17 (range 920). Median total $A B$ use was $1285 \mathrm{DDD} / 1000$ pd (range 639-2393). The fraction of the most used $A B$ group ranged from 13 to $41 \%$ of total $A B$ use (median 23\%). In the multivariable analysis the group ADs of third generation (3G) cephalosporines, macrolides and methicillin resistant staphylococcus aureus (MRSA) active $A B$, respectively, were significantly associated with the total $A B$ use in the logistic regression model. Interestingly, none of the structural parameters showed a significant association with total $\mathrm{AB}$ use.

\section{Conclusion}

Diversity of AB usage is great among German ICUs, but structural parameters do not seem to determine significantly the extent of total $A B$ use. Rather, usage of certain $\mathrm{AB}$ groups (3G cephalosporines, macrolides and MRSA active substances) seems to drive the overall $A B$ consumption. However, whether this is due to certain patient characteristics or at least partially to imprudent $A B$ use cannot be answered by our calculations alone and should be further investigated by complementary methods.

\section{Disclosure of interest}

None declared.

Published: 20 June 2013

doi:10.1186/2047-2994-2-S1-050

Cite this article as: Schneider et al: 0050: Antibiotics in 70 German

intensive care units: risk factors for high overall consumption.

Antimicrobial Resistance and Infection Control 2013 2(Suppl 1):050.

Hygiene and Environmental Medicine, Charite, Berlin, Germany 